\title{
Preface
}

\section{The 'Gianni Benzi' Pharmacological Research Foundation initiatives}

\author{
Donato Bonifazi \\ Gianni Benzi Foundation, Via Abate Eustasio 30, 70010, Valenzano, BA, Italy \\ E-mail: info@benzifoundation.org
}

The 'Gianni Benzi' Pharmacological Research Foundation is a registered "notfor-profit" Scientific Research Organisation, founded on November 9th, 2007. The mission of the Foundation is to contribute to the advancement of pharmacological research in those fields where the scientific heritage of Prof. Benzi, is more prominent, as well as to the promotion of innovative drugs for all European citizens in the context of a competitive and advanced European Pharmaceutical System and to the safety of drugs and the fight against the drug abuse and misuse, including doping.

Since Prof. Benzi was for many years member of the EMEA Board and the COMP, the Foundation retains a particular attention to the implementation of a valid Advanced European Pharmaceutical System as a system capable of making a significant contribution to the improvement of the quality of treatment and access to pharmaceutical products for all citizens, with particular attention paid to the more disadvantaged categories.

One of the main initiatives in this field is represented by the organisation of the annual 'Foresight Training Courses', short courses for groups of experts, companies, regulatory bodies and researchers involved in drug development activities. The courses are related to, and aim to complement and support, existing training experiences in the field in Italy and in Europe (Masters, High Level Courses, etc.) in view of the extremely high complexity to which this system operates. In fact, this is a complex setting in which medical connotations are compared and integrated with legal, economic and regulatory elements. In particular, experts in Regulatory Affairs must face the challenges of human research, with the development of new advanced therapies (gene and cell therapy), the uncertainties and problems of safety, the increasing cost of the research, and the economic pressure on Public Health services which require the implementation of new cost-efficacious measures.

The courses also aim to involve participants from many European Countries, including countries which have recently joined the EU, to promote their integration into the European regulatory context and are held in English according to an interdisciplinary approach. The teaching staff are selected on the basis of their documented skills and expertises. 
The First Foresight Training Course was held in Pavia, in September 2008 and was focused on the MA Centralised European Procedures and the Paediatric Regulation.

The Second Foresight Training Course, in line with other Gianni Benzi Foundation's initiatives promoting pharmacological research and innovation, was held in Pavia too in 2009 and dealt with the most innovative regulatory issues developed during the last years, particularly in the area of Advanced Therapies, Orphan Drugs and Paediatric Medicines.

The new centralised procedure for the registration of Advanced Therapy Medicinal Products (ATMPs) was analysed during the course, as well as the entire procedural process that leads to the registration of an Orphan Drug (OD). A special session was dedicated to innovation in the paediatric medicines field introduced by the recently approved European Paediatric Regulation.

The Second Foresight Training Course was organised in collaboration with the Master of Regulatory Science 'Gianni Benzi' held at Pavia's University and with TEDDY, the Task Force in Europe for Drug Development in the Young, a FP6 funded project aimed to the development of good quality medicines studied and tailored for children, SIAR (Italian Society for Regulatory Affairs) and MEGRA (Mittel Europäische Gesellschaft für Regulatory Affairs).

Key representatives from EMA, from the Italian Medicines Agency (AIFA) and from other National Agencies, together with other experienced speakers from academy, public bodies and pharmaceutical industries participated and contributed to the Course. 